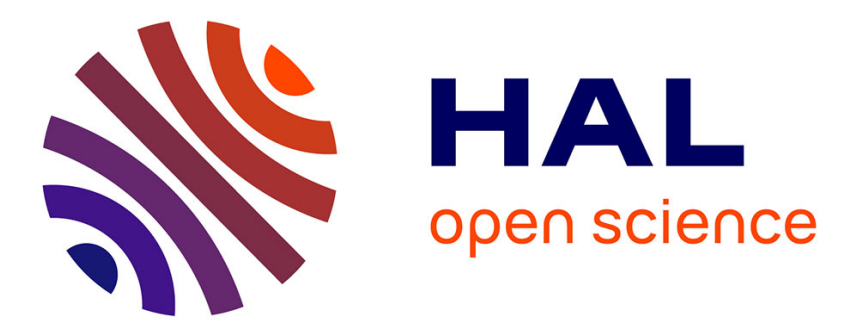

\title{
Optical flow focusing: Light-induced destabilization of stable liquid threads
}

Matthieu Robert de Saint Vincent, Hamza Chraibi, Jean-Pierre Delville

\section{To cite this version:}

Matthieu Robert de Saint Vincent, Hamza Chraibi, Jean-Pierre Delville. Optical flow focusing: Lightinduced destabilization of stable liquid threads. Physical Review Applied, 2015, 4 (4), pp.044005 (1-8). 10.1103/PhysRevApplied.4.044005 . hal-01198761

\section{HAL Id: hal-01198761 https://hal.science/hal-01198761}

Submitted on 16 Sep 2015

HAL is a multi-disciplinary open access archive for the deposit and dissemination of scientific research documents, whether they are published or not. The documents may come from teaching and research institutions in France or abroad, or from public or private research centers.
L'archive ouverte pluridisciplinaire HAL, est destinée au dépôt et à la diffusion de documents scientifiques de niveau recherche, publiés ou non, émanant des établissements d'enseignement et de recherche français ou étrangers, des laboratoires publics ou privés.

\section{다(1)(2)}

Distributed under a Creative Commons Attribution - ShareAlikel 4.0 International 


\title{
Optical flow focusing: Light-induced destabilization of stable liquid threads
}

\author{
Matthieu Robert de Saint Vincent, ${ }^{1,2, *}$ Hamza Chraïbi, ${ }^{2}$ and Jean-Pierre Delville ${ }^{2, \dagger}$ \\ ${ }^{1}$ IPR, UMR CNRS 6251, Campus Beaulieu, Université Rennes 1, 35042 Rennes, France \\ ${ }^{2}$ University of Bordeaux, CNRS, Laboratoire Ondes et Matière d'Aquitaine, UMR 5798, F-33400 Talence, France
}

(Dated: September 11, 2015)

\begin{abstract}
Confinement of flowing liquid threads by solid walls makes them stable with respect to the Rayleigh-Plateau instability. We demonstrate here that light can break this stability, by forcing locally the deformation of the liquid interface through thermally-induced Marangoni stresses. Depending upon the confining conditions and fluid properties, this optocapillary deformation either pinches or inflates the thread, which may in both cases lead to its localized fragmentation into droplets. In the pinching regime, the laser beam behaves as a wall-free constriction that flow focuses the thread, leading to successive regimes of single and multiple periodicity. Light-driven local Marangoni stresses may prove an elegant contactless alternative to control reversibly the thread-todroplet transition for digital microfluidics.
\end{abstract}

PACS numbers: 47.55.dm, 47.20.Dr, 47.55.db

\section{INTRODUCTION}

Liquid threads or jets [1] have been shown naturally unstable since the seminal work of Savart [2], later clarified by Plateau [3] and Lord Rayleigh [4-6], who identified the minimization of surface energy as the driving mechanism. By geometrically constraining the volume available for surface deformation, rigid boundaries slow down their development, improving stability [7-10] up to full stabilization when the thread is squeezed [11]. Thus, liquid threads are either unstable (convectively or absolutely [10]) when they are three dimensional, or absolutely stable when behaving as ribbons, depending on the confinement.

This confinement-induced stability is a key issue in two-phase microfluidics [12]. On the one hand, droplet production from thread instability, also called digital microfluidics, is essential for a considerable number of processes involving droplets from micro-chemistry and physical chemistry to biotechnologies [13], or ink-jet printing [14]. On the other hand, stable co-flows are more efficient to transport fluids over long distances due to the reduction of the friction compared to a confined droplet flow. Consequently, it became crucial for lab-on-a-chip applications to trigger the thread-to-droplet instability at well-defined locations and produce droplets of size appropriate to the application. The first approach consisted in designing the geometry of the contact between the immiscible fluid streams coming from different channels [15]. Three main designs were investigated: T-junctions [16], axial co-flows [10] and flow-focusing through a constriction [17]. The drop size can be actuated using the size and the shape of the channels, the inlet flow rates and the interfacial properties between the immiscible liquids. However, while extremely reliable, some applications require versatility in flow and droplet management which

\footnotetext{
* rdsv.matthieu@gmail.com

$\dagger$ jean-pierre.delville@u-bordeaux.fr
}

cannot be fulfilled with a rigid design of the chip and the unavoidable transient regimes when changing flow characteristics [18]. Active control of the thread instability was thus proposed to tune the drop size in flow focusing and T-junctions with mechanical [19, 20], electrical $[21,22]$, acoustical $[23,24]$ or optical breakdown [25] actuation, to cite a few.

The step forward consists in controlling reversibly the thread-to-droplet transition in confined geometries to either convey stable viscous co-flows or dispense droplets on demand and at any location on a chip. This issue has already been explored using UV exposure of specific photosensitive cis-trans surfactants [26], or DC electric fields to form Taylor cones on droplets and trigger the droplet-to-thread transition at high voltage [27], both approaches being dedicated to lab-on-chip applications and requiring specific fabrications or responsive chemicals. In the present investigation we address this question considering a totally different situation in the sense that our approach does not require dedicated microfabrication or specific surfactant other that those classically used in microfluidics. As surface tension depends on temperature, a thermal gradient sets a surface tension gradient and therefore produces tangential stresses along the interface separating two immiscible fluids [28]. These Marangoni stresses in turn induce interfacial flows and may cause interface deformations $[29,30]$. Thermal modulations of surface stresses were actually employed on free-surface threads to force a specific mode of the Rayleigh-Plateau instability or conversely to oppose to the most unstable mode and delay the instability onset [31-33].

We demonstrate experimentally and numerically that optocapillarity, i.e. light-driven Marangoni stresses, can deform and eventually destabilize stable liquid threads, as conceptually illustrated on Fig. 1. Lasers are indeed very appealing to trigger thermocapillary flows because optical focusing in absorbing liquids yields very large thermal gradients for a given overheating [34, 35]. Using a 1D model, we first illustrate that the interfacial deformation induced by a thermal gradient nonlinearly 


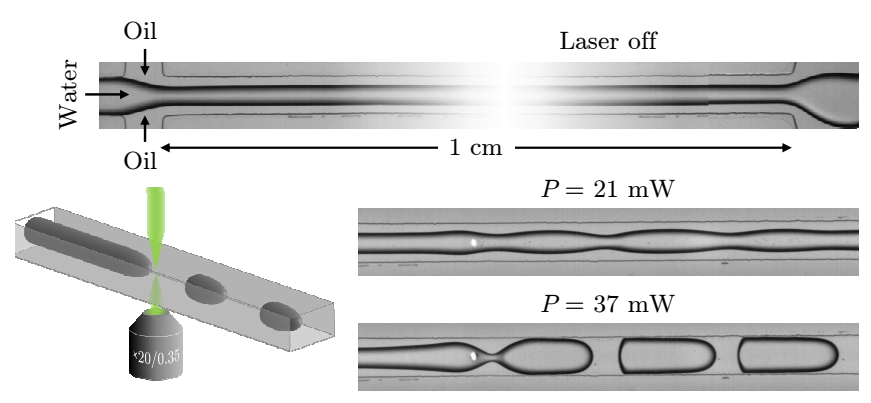

Figure 1. (Color online) Concept of optical flow focusing: A laser beam focused onto a stable liquid thread flowing within a microfluidic channel (top image) can destabilize it (middle image) and reversibly force its fragmentation (bottom image). The white dot corresponds to the location of the laser beam.

depends on both the viscosity and the confinement of the liquid layers involved. This is then used to demonstrate how optocapillarity can locally pinch, and more surprisingly inflate, a confined liquid thread, and trigger instability despite the confinement. We finally show that optocapillary pinching behaves as a wall-free tunable flow focusing which controls the thread fragmentation into droplets regimes of different periodicity reminiscent of various nonlinear systems [36-42].

\section{OPTOCAPILLARY DEFORMATION OF LIQUID SHEETS AND THREADS}

The interface deformation of confined threads driven by Marangoni stresses can be unveiled with a simple three-layer 1D model. A layer of liquid 1, of height $2 \mathrm{H}_{1}$, is sandwiched between two immiscible layers of liquid 2, of height $H_{2}$ each. We solve the Stokes equation, $-\boldsymbol{\nabla} p_{i}+\eta_{i} \nabla^{2} \boldsymbol{u}_{i}+\rho_{i} \boldsymbol{g}=\mathbf{0}$, in each phase $(i=1,2)$, in the upper half-system as sketched on Fig. 2 (a). Here $p$ and $\boldsymbol{u}$ denote the pressure and velocity fields, $\boldsymbol{g}$ is the gravitational acceleration and $\rho$ and $\eta$ are the liquid density and viscosity, respectively. We assume incompressible liquids with a lubrication approximation, and set the following boundary conditions: (i) $u_{x, 2}\left(z=H_{2}\right)=0$ (no slip at the wall), (ii) $\left(\mathrm{d} u_{x, 1} / \mathrm{d} z\right)_{z=-H_{1}}=0$ (by symmetry), and (iii) $u_{x, 1}(z=0)=u_{x, 2}(z=0)$ (continuity). In addition, the incompressibility imposes, in a finite system, $\int_{-H_{1}}^{0} u_{x, 1}(z) \mathrm{d} z=\int_{0}^{H_{2}} u_{x, 2}(z) \mathrm{d} z=0$. This condition is imposed either by the remote vertical boundary of the system, or by mirror symmetry of the interfacial tension gradient along the $x$ axis, as in our experiment. This bounding of the system creates a deep layer counterflow as sketched on Fig. 2 (a) that prevents any net flow through either phase.

An interfacial tension gradient, such as $\mathrm{d} \sigma / \mathrm{d} x=$ $\eta_{1}\left(\mathrm{~d} u_{x, 1} / \mathrm{d} z\right)_{z=0}-\eta_{2}\left(\mathrm{~d} u_{x, 2} / \mathrm{d} z\right)_{z=0}$, drives interfacial flows in both phases, that deform the interface of profile $h(x)$. Solving the Stokes equation for $\mathrm{d} h / \mathrm{d} x \ll 1$

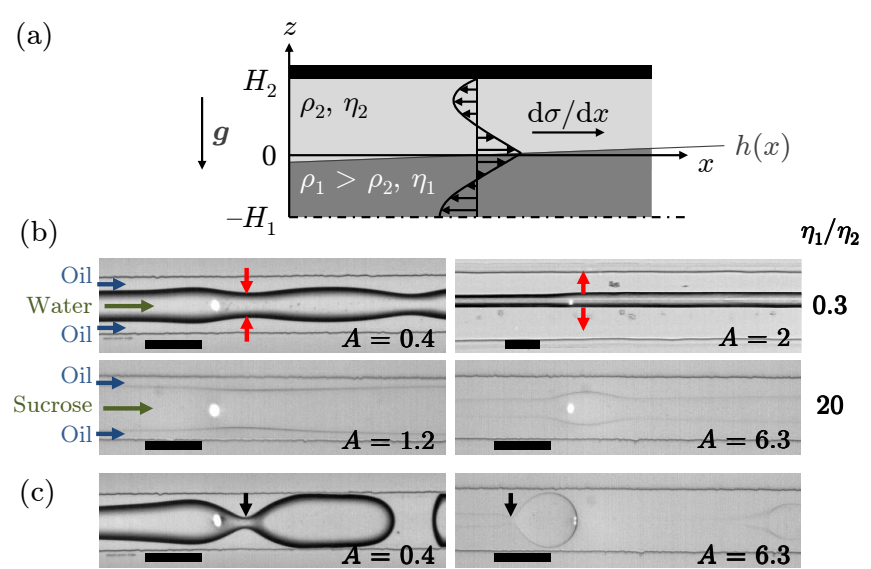

Figure 2. (Color online) (a) Sketch of the three-layer 1D model system; only the upper half is represented. (b) Reversal of the optocapillary deformation when varying the viscosity ratio or the confinement. Thick threads $(A<1)$ pinch while thinner ones $(A>1)$ rather bulge as indicated by the red arrows. (c) For large enough power, the thread eventually breaks in either case. The black arrows indicate the breakup location. Flow rates and laser power $\left(Q_{1}, Q_{2} ; P\right)$ in $\mu \mathrm{L} \mathrm{min}{ }^{-1}$ and $\mathrm{mW}$ are respectively $(2,1 ; 21),(0.2,0.2 ; 13),(1.5,2 ; 36)$, $(0.03,0.2 ; 12),(2,1 ; 37)$ and $(0.03,0.2 ; 18)$ from top to bottom; the white dot is the laser spot location observed by the fluorescence of the water-dye solution. Scale bar, $100 \mu \mathrm{m}$.

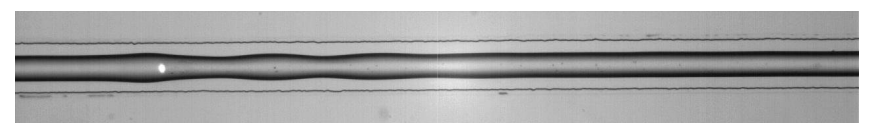

Video 1. Movie illustrating the pinching deformation of a confined liquid thread under continuous laser forcing. The thread does not break; the pinching deformation advects and decays after $3-4$ wavelengths. The real-time dynamics is ten times faster; $P=27 \mathrm{~mW},\left(Q_{1}, Q_{2}\right)=(3,1.5) \mu \mathrm{L} \mathrm{min}^{-1}$.

with the above assumptions yields

$$
\frac{\mathrm{d} h}{\mathrm{~d} x}=\frac{\ell_{\mathrm{c}}^{2}}{H_{1} H_{2} \sigma_{0}} \frac{\eta_{1} H_{2}^{2}-2 \eta_{2} H_{1}^{2}}{\eta_{1} H_{2}+4 / 3 \eta_{2} H_{1}} \frac{\mathrm{d} \sigma}{\mathrm{d} x}
$$

with $\ell_{\mathrm{c}}=\sqrt{\sigma_{0} /\left(\rho_{1}-\rho_{2}\right) g}$ the capillary length. The deformation at the point of largest interfacial tension should thus be oriented from liquid 1 to liquid 2 if $\left(\rho_{1}>\rho_{2}\right)$

$$
A=\frac{H_{2}}{H_{1}} \sqrt{\frac{\eta_{1}}{2 \eta_{2}}}>1 .
$$

For a given interfacial stress, the direction of the deformation is thus determined by the relative thicknesses and viscosities of the fluid layers. Note finally that the amplitude and orientation of the interfacial tension gradient do not influence the basic mechanism of interfacial deformation; reversing the sign of the interfacial tension gradient should thus only reverse the orientation of the deformation.

We force optocapillary deformation of confined waterin-oil threads into PDMS microfluidic channels [43]. Water $\left(\eta_{1}=1 \mathrm{mPas}\right)$ and hexadecane $\left(\eta_{2}=3.3 \mathrm{mPas}\right)$ are 
injected at constant flow rates $\left(Q_{1}\right.$ and $Q_{2}$, respectively), meet at a cross-shaped junction, and co-flow alongside further downstream. The rectangular cross-section of the channel (typically, 100- $\mu \mathrm{m}$ wide, $50-\mu \mathrm{m}$ high) ensures a vertical confinement that increases thread stability [10]. To favor thread formation at low flow rates, the interfacial tension is reduced by adding two surfactants, sorbitan monooleate (Span 80, 0.3\% w/w) in oil and sodium dodecyl sulfate (SDS, $2.5 \% \mathrm{w} / \mathrm{w}$ ) in water, both above the critical micelle concentration (CMC). The equilibrium interfacial tension is $\sigma_{0} \sim 10^{-4} \mathrm{~N} \mathrm{~m}^{-1}$ [44]. Appropriate choices of flow rates thus produce stationary water-in-oil threads, stable during minutes to hours over the whole length of the channel (1 cm, see Fig. 1); the weak vertical squeezing allows to consider a circular cross-section of diameter $2 R_{0}$ similar to the channel height.

Optocapillary deformations were achieved by focusing a continuous $\mathrm{TEM}_{00} \mathrm{Ar}^{+}$laser (wavelength in vacuum $\left.\lambda_{0}=514.5 \mathrm{~nm}\right)$ on the centerline of the flow as illustrated on Fig. 2 (b,c). The laser position was sufficiently far from the junction $(0.8 \mathrm{~mm})$ to assume parallel flow. A dye, uranine (disodium fluorescein), is added in water $(0.1 \% \mathrm{w} / \mathrm{w})$ to ensure heating occurs by light absorption [35]. A $\times 20$ microscope objective both focuses the beam onto the liquid interface (beam waist $\omega_{0}=1.3 \mu \mathrm{m}$ ), and images the flow in the channel. Finally, the deformation dynamics was recorded by a CMOS fast camera (Lightning RTD 16000).

Using surfactants above the CMC may reverse the direction of thermocapillary flows compared to classical cases, most likely due to subtle couplings between thermal and molecular transport of surfactants along the interface [45]. This is accounted for by introducing an 'effective' coefficient of variation of interfacial tension with temperature, $(\partial \sigma / \partial T)_{\text {eff }}$, that turns out to be positive in this case. The point of highest interfacial tension therefore coincides with the hot spot.

Continuously shining the thread at moderate laser power deflects the interface as represented on Fig. 2 (b). From Eq. 2, one expects thick (resp. thin) threads to pinch (resp. bulge), the transition being governed by the ratio of viscosities. We first varied the cross section of a water-in-hexadecane thread via the flow rates, as shown on the left and right images of Fig. 2 (b), top row. Pinching occurs when $H_{1} / H_{2} \simeq 1.1$ (left, $A=0.4$ ), while a thinner thread $\left(H_{1} / H_{2} \simeq 0.2\right)$ slightly humps (right, $A=2$ ). We then changed the viscosity ratio by adding sucrose in water $\left(60 \% \mathrm{w} / \mathrm{w}, \eta_{1} \simeq 60 \mathrm{mPas}\right)$. Similar behavior is seen when changing the liquid thickness ratio, as illustrated on the bottom row of Fig. 2 (b) $\left[H_{1} / H_{2} \simeq 2.5\right.$ (left, $A=1.2$ ) and 0.4 (right, $A=6.3$ )]. Figure $2(\mathrm{~b})$ also shows the limitations of the $1 \mathrm{D}$ model close to the transition value $A=1$ : as expected, large powers are necessary for interface deformation but a bulge was anticipated at $A=1.2$ instead of a weak pinching.

Moreover, for pinched water threads, the deformation propagates downstream, leading to subsequent modula-
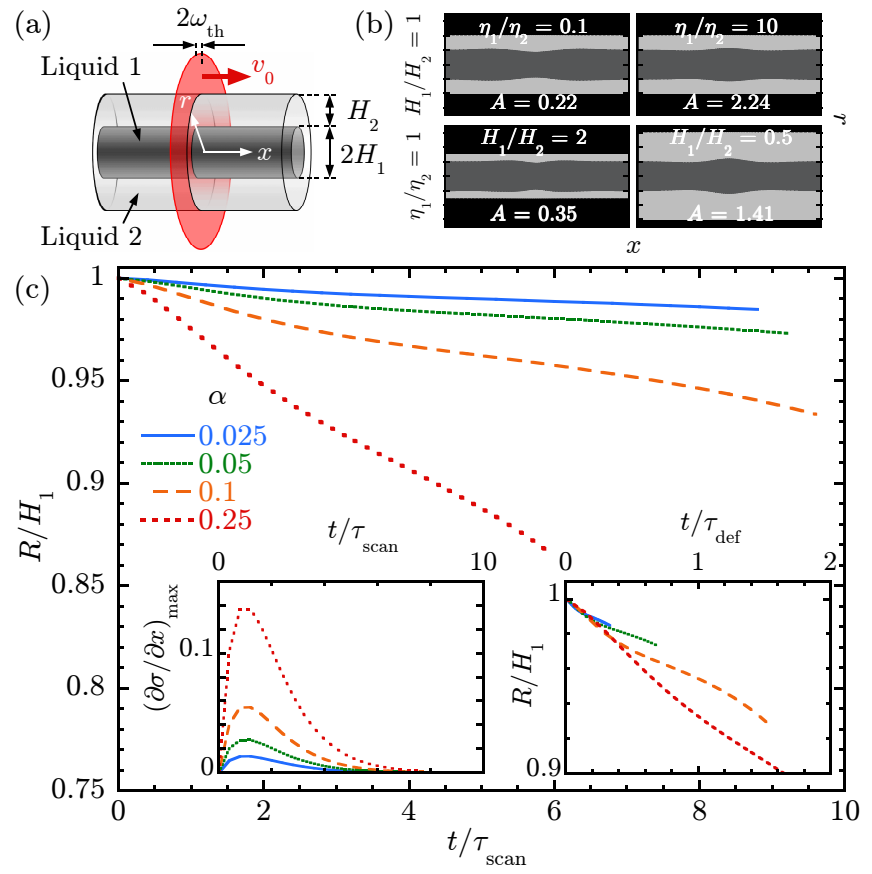

Figure 3. (Color online) (a) Sketch of the simulated geometry. (b) Longitudinal profile of the thread interface when varying the viscosity ratio and the degree of confinement; $\alpha=0.25, t / \tau_{\text {def }} \simeq 3$ except for $A=0.35\left(t / \tau_{\text {def }} \simeq 6\right)$. (c) Pinching dynamics for four values of $\alpha$, with $\eta_{1} / \eta_{2}=0.1$ and $H_{1} / H_{2}=3.33$, in response to an interfacial stress $\partial \sigma / \partial x$ evolving as represented in Left Inset. Right Inset: Same dynamics, normalized by $\tau_{\text {def }}$.

tions that decay within 3-4 wavelengths (see Video 1 and Fig. 1, middle image). This observation illustrates the absolute stability of the confined thread. Increasing the laser power may actually break the thread periodically, producing consecutive droplets. Surprisingly, this process occurs for either pinched or bulged threads [Fig. 2 (c)]. In the former case, the fragmentation is directly initiated near the laser spot when the pinched neck becomes unconfined [46]. The mechanism in the latter case is more subtle. Starting from the continuous thread, some liquid accumulates into the growing bulge, that partly obstructs the channel. The resulting overpressure forces breakup upstream of the hot spot [47]. Once broken, the upstream advancing front of the thread is blocked by the laser [35], allowing another droplet to grow and eventually break upstream through the same process.

\section{PINCHING DYNAMICS}

The reversal of the optocapillary deformations can be well understood by the effect of confinement on viscous liquid layers. We first use the Boundary Element Method [48] to perform numerical simulations on a stationary thread (initially cylindrical), surrounded by an axisymmetric liquid shell as represented on Fig. 3 (a). A 
Gaussian heating sheet, of transverse profile $\Delta T(r, x) \sim$ $\mathrm{e}^{-2 x^{2} / \omega_{\text {th }}^{2}}$ and width $\omega_{\text {th }}=2 H_{1}$, imposes a transient interfacial stress $\partial \sigma / \partial x \sim \Delta \sigma t \mathrm{e}^{-t / \tau_{\text {scan }}}$ in a cross-sectional plane $\left[\Delta \sigma=(\partial \sigma / \partial T)_{\text {eff }} \Delta T\right]$. Defining $\tau_{\text {scan }}=2 \omega_{\mathrm{th}} / v_{0}$, this form reproduces the temperature field produced by a laser beam scanned at the velocity $v_{0}$ [49]. Considering the tangential stress condition at the interface between liquids 1 and 2, we also define a characteristic deformation time, $\tau_{\text {def }}$, as

$$
\tau_{\text {def }}=\frac{\eta \omega_{\mathrm{th}} H_{1}}{H_{2} \Delta \sigma}
$$

Figure 3 (b) shows for $\alpha=\Delta \sigma / \sigma_{0}=0.25$ that the reversal behavior of deformations versus thicknesses and viscosities, obtained both experimentally (Fig. 2) and theoretically in $1 \mathrm{D}$ (Eq. 2), is retrieved numerically. Moreover, Fig. 3 (c) depicts the pinching dynamics [evolution of the minimal thread radius, $R(t)$ ] in response to the time-dependent interfacial stress for four different $\alpha$ [Fig. 3 (c), left inset]. Simulations were performed in a confined case, $H_{1} / H_{2}=3.33$, and $\alpha$ values were chosen so as to investigate the influence of transient heating effects on optocapillary deformations (typically $\left.\tau_{\text {scan }} / \tau_{\text {def }} \in[0.1,1]\right)$. Not surprisingly, the stronger the stress, the faster the deformation; note that the final acceleration is due to the Rayleigh-Plateau instability because the simulated threads are not absolutely stabilized by the confining walls. The right inset of Fig. 3 (c) finally shows the pertinence of $\tau_{\text {def }}$ to capture the initial dynamics of interface deformation as the pinching dynamics obtained at different $\alpha$ collapse onto a single behavior in reduced time at first stage. The deformation then slows down as the heating decays, and the Rayleigh-Plateau instability finally develops.

However, as experiments are performed on flowing threads, we compare $\tau_{\text {def }}$ with the other time scales involved: heating time $\tau_{\text {heat }}=\omega_{\mathrm{th}}^{2} / D$, viscous time $\tau_{\text {visc }}=\rho R_{0}^{2} / \eta$, capillary time $\tau_{\text {cap }}=\rho v_{0} R_{0}^{2} / \sigma_{0}$, and advective time $\tau_{\mathrm{adv}}=R_{0} / v_{0}$. Assuming a temperature distribution as calculated in Ref. [35], we find $\omega_{\text {th }} \simeq 5 \mu \mathrm{m}$ for $\omega_{0} \simeq 1.3 \mu \mathrm{m}$. We choose $\eta=\left(\eta_{1}+\eta_{2}\right) / 2$ the average viscosity; $D \simeq 10^{-7} \mathrm{~m}^{2} \mathrm{~s}^{-1}$ is the thermal diffusivity, and $v_{0}$ is the initial velocity of the thread. For waterhexadecane pinching threads, we estimate $\tau_{\text {heat }} \simeq 0.25$ $\mathrm{ms}, \tau_{\text {visc }} \simeq 0.3 \mathrm{~ms}, \tau_{\text {cap }} \sim 0.05 \mathrm{~ms}$, and $\tau_{\text {adv }} \simeq 2-5 \mathrm{~ms}$.

Furthermore, due to the vertical confinement, the deformation rate of the flowing thread is limited by the draining of the outer fluid through lubrication films of initial thickness $\varepsilon$ imposed by the disjoining pressure. The deformation time therefore becomes $\tau_{\text {def }}=$ $\eta_{2} \omega_{\mathrm{th}} R_{0} /(\varepsilon \Delta \sigma)$. Assuming $(\partial \sigma / \partial T)_{\mathrm{eff}}$ in the order of $10^{-5} \mathrm{~N} \mathrm{~m}^{-1} \mathrm{~K}^{-1}$, a temperature increase of $1 \mathrm{~K}$ per $\mathrm{mW}$ [35], and setting $\varepsilon \sim 300 \mathrm{~nm}$ [50], we estimate $\tau_{\text {def }} \sim 2-$ $40 \mathrm{~ms}$ in the power range investigated. From these time scales $\left(\tau_{\text {heat }}, \tau_{\text {cap }}, \tau_{\text {visc }} \ll \tau_{\text {def }}\right)$, we conclude that the thermocapillary deformation of the confined thread mainly competes with its advection by the flow.

We experimentally investigate this interplay by mea-

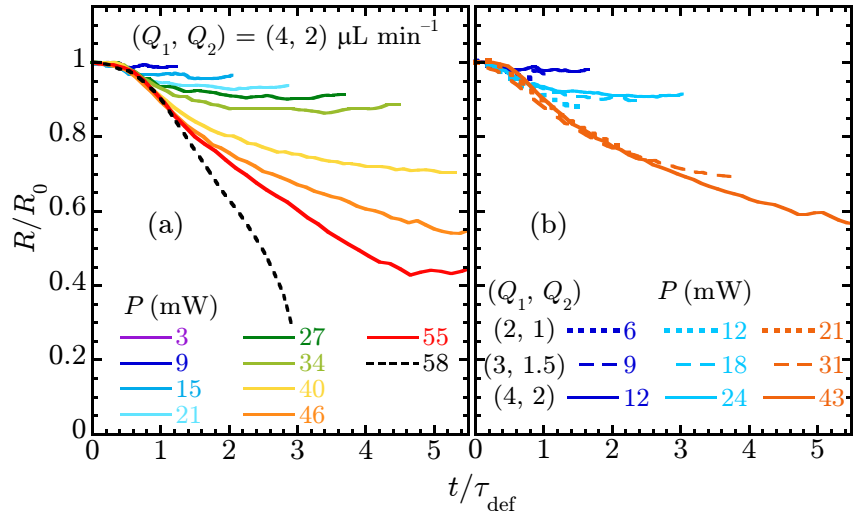

Figure 4. (Color online) Pinching dynamics of a stable thread: normalized neck radius $R / R_{0}$ versus reduced time $t / \tau_{\text {def }}$. (a) Fixed thread velocity $\left(v_{0} \simeq 1 \mathrm{~cm} \mathrm{~s}^{-1}\right)$, increasing laser powers. Dashed line: thread pinching up to rupture. (b) Comparison of the pinching dynamics for the three flow rate couples investigated, for three laser powers selected accordingly.

suring the pinching dynamics in response to a stepshaped, 16.7-ms wide laser pulse. The temporal evolution of the minimal thread radius is represented in dimensionless terms on Fig. 4 (a) for laser powers ranging from 3 to $58 \mathrm{~mW}$ and a fixed thread velocity $\left(v_{0} \simeq 1 \mathrm{~cm} \mathrm{~s}^{-1}\right)$. Up to $55 \mathrm{~mW}$, the deformation initially increases, following a common behavior, then saturates before the thread has become unstable and finally attenuates. The larger the beam power, the later the deviation from the common trend. The collapse of all initial dynamics in reduced time, consistent with simulations, emphasizes that the tangential stresses drive the first stages of the deformation in presence of confinement. As suggested by the estimated time scales, the later saturation should result from the advection of the deformation at longer time as the thread flows. We therefore varied the flow rates $Q_{1}$ and $Q_{2}$, keeping their ratio constant so the initial confinement remains unchanged. Figure 4 (b) depicts nine dynamics, gathered into three groups of constant $\tau_{\text {adv }} / \tau_{\text {def }} \sim P / v_{0}$ ratio - higher ratio yielding to more pronounced deformations and a delayed deviation from the common pinching behavior. In each group, the three dynamics overlap quite well, supporting that the pinching dynamics of a stable flowing thread is controlled by the interplay between optocapillarity and the fluid advection.

\section{THREAD FRAGMENTATION BY OPTICAL FLOW FOCUSING}

Above a threshold power $[P \geqslant 58 \mathrm{~mW}$ in Fig. 4 (a)], the deformation amplifies, and the pinching follows a specific dynamics leading to the thread breakup [44]. The thread indeed becomes locally unstable when the neck narrows down to a diameter significantly smaller than the smallest length of the channel, here its height [46]. This rupture occurs slightly downstream of the laser spot, and 


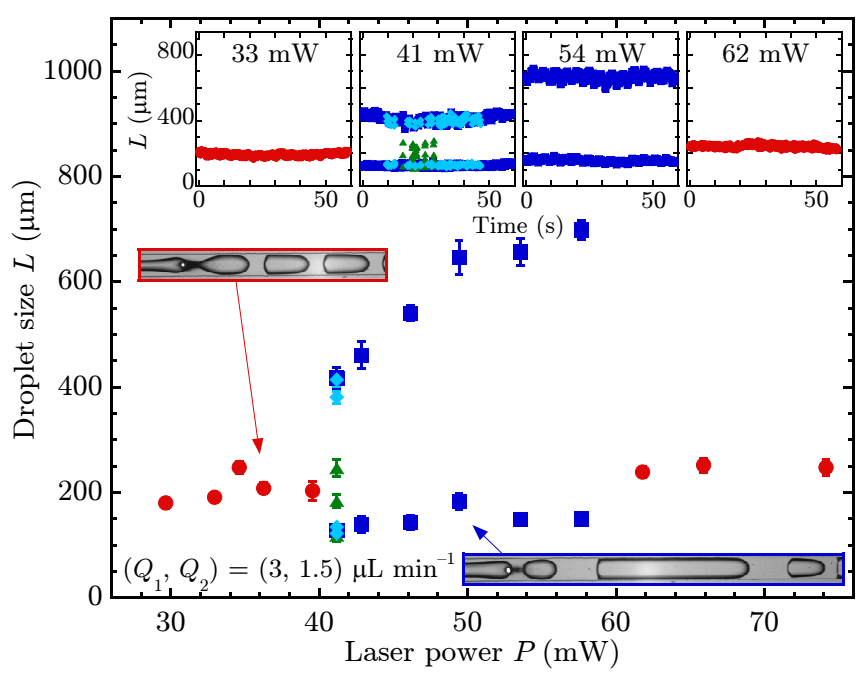

Figure 5. (Color online) Bifurcation diagram: period-one (red circles), period-two (dark blue squares), period-three (green triangles), period-four (light blue diamonds) droplet production patterns. Error bars are \pm 1 standard deviation. Insets: examples of sequences measured over one minute.

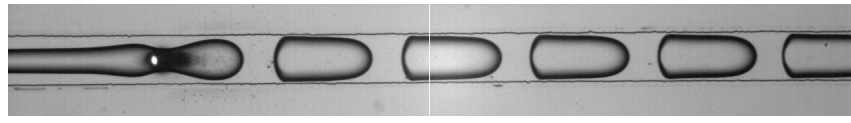

Video 2. Movie illustrating the period-1 fragmentation of a confined liquid thread under moderate continuous laser forcing. The thread interface never touches the vertical walls. The real-time dynamics is ten times faster; $P=36 \mathrm{~mW}$, $\left(Q_{1}, Q_{2}\right)=(3,1.5) \mu \mathrm{L} \mathrm{min}^{-1}$.

releases droplets as represented on the left image of Fig. 2 (c). Note however that the thread remains continuous upstream of the laser spot, the optocapillary pinching behaving as a wall-free tunable flow focusing which locally forces the thread fragmentation.

To investigate this optical flow focusing effect, we characterized under continuous forcing exposure and over long times (up to several minutes [18]) the sequences of droplets emitted on a wide power range. Distinct emission patterns emerge depending on the laser power (Fig. 5). At low power (below $40 \mathrm{~mW}$ ), the droplet production is simply periodic, period-1, resulting in streams of highly monodisperse droplets (left inset). In an intermediate power range (here, between 40 and $60 \mathrm{~mW}$, second and third insets), two distinct droplets, one long and one short, are successively produced. This period-2 regime may coexist close to the bifurcation (especially, at $P=41 \mathrm{~mW}$ ) with more complex transient regimes, involving three (one short, one intermediate, one long) or four droplets (two distinct period-2 sequences entangled) per sequence. Finally, further increasing the laser power (above $60 \mathrm{~mW}$ ) brings the system back to a period-1 regime (right inset).

The alternation between sequences of single and mul-

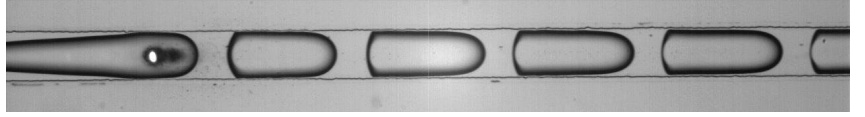

Video 3. Movie illustrating the period-1 fragmentation of a confined liquid thread under strong continuous laser forcing. The thread interface permanently touches the vertical walls. The real-time dynamics is ten times faster; $P=62 \mathrm{~mW}$, $\left(Q_{1}, Q_{2}\right)=(3,1.5) \mu \mathrm{L} \mathrm{min}-1$.

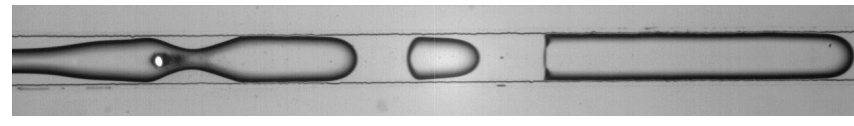

Video 4. Movie illustrating the period-2 fragmentation of a confined liquid thread under continuous laser forcing. The thread interface alternatively does and does not touch the lateral walls. The real-time dynamics is ten times faster; $P=$ $54 \mathrm{~mW},\left(Q_{1}, Q_{2}\right)=(3,1.5) \mu \mathrm{L} \min ^{-1}$.

tiple periodicities results from the geometric confinement of the thread near the breakup point. As shown on the left panel of Fig. 2 (b,c), the liquid expelled from the pinched area accumulates both upstream and downstream. The water thread may therefore touch the lateral walls and obstruct the channel upstream, altering the pinching dynamics due to the slow draining through the outer oil films. More specifically, in the low- and highpower period-1 regimes, the widest part of the thread after breakup respectively never and permanently touches the wall (Videos 2 and 3). Thus, in both cases, boundary conditions do not vary during the pinching, resulting in periodic droplet emission. In contrast, in the regime of higher periodicity the bulged part of the thread strongly evolves during a full sequence, alternatively touching (when a long droplet forms) and not touching (hence releasing a short droplet) the wall (Video 4). This nonlinear behavior is reminiscent of feedback mechanisms occurring either at the leading edge of a thread, as in the widely-studied dripping faucet $[36,37]$ or into microfluidic constrictions $[39,40]$. The simplified period1-period-2-period-1 bifurcation diagram observed here results from the interplay between the pinching and the obstruction due to the channel confinement. Moreover, contrary to classical flow focusing cases when inertia may be crucial, optical flow focusing allows to control the drop formation at low Reynolds $\left(\operatorname{Re}=\tau_{\text {visc }} / \tau_{\text {adv }} \sim 10^{-1}\right)$ and Weber $\left(\mathrm{We}=\tau_{\text {cap }} / \tau_{\text {adv }} \sim 10^{-2}\right.$ ) numbers.

\section{CONCLUDING REMARKS}

In this investigation, we have demonstrated that optocapillarity, i.e. light-driven local Marangoni stresses, can trigger the capillary instability of confinement-stabilized liquid threads. We demonstrated that depending on the interplay between the fluid confinement and viscosities the optocapillary instability forces either bulging or 
pinching of the confined thread and local breakup into droplets above a critical Marangoni stress. In the bulging case, the breakup of the thread occurs upstream of the laser hot spot due to Marangoni flow-induced accumulation leading to partial obstruction of the channel; the resulting overpressure forces breakup. In optocapillary pinching, the fragmentation is directly initiated near the laser spot when the pinched neck becomes locally unconfined. Optocapillary pinching thus behaves as a wall-free flow focusing device that can be used to control and finetune the thread-to-droplet transition while leaving infusing flow rates constant. We also showed numerically and experimentally that the early stage pinching dynamics obtained for different flow and heating conditions collapse onto a single behavior when time is reduced with a characteristic deformation time. Finally, we investigated the efficiency of this new optical flow focusing device by characterizing the sequences of droplets produced under continuous optical forcing and over long periods of time. At low Marangoni stress the droplet production is period-1 with a high monodispersity. At intermediate Marangoni stresses, a period-2 bifurcation emerges with two distinct droplets. Finally, further increase of the stress brings the droplet emission back to a period1 regime. This bifurcation by period doubling, which is quite common in dripping, results here from the interplay between the optical pinching and the channel obstruction due to Marangoni flows. Two important clues may be raised for applications. Contrary to classical flow focusing situations, even in microfluidics, no inertia is required to produce drops optically; optical flow focusing thus offers an alternative at low Reynolds and Weber numbers. Then, the period-2 regime, and strictly speaking any $n$ period regime with $n \geqslant 2$, are generally not desirable for applications as the drop size usually represents the ele- mental digit for quantitative experiments (micro-reactor in chemistry, final dot in ink-jet printing, etc.). Nonetheless, fast droplet production requires large flow rates and at the same time the resulting inertia may alter the final drop distribution. Our experiment shows that although optical flow focusing does not prevent droplet production from bifurcation, finite-size effect due the confinement reduces the parameter extension of the bifurcation region and then preserves monodispersity over very large variations of control parameters. As a final remark, the efficiency of optical flow focusing requires interface deformation and then co-flows at low interfacial tensions. We overcome this difficulty by adding two surfactants, a non-ionic (resp. ionic) one in oil (resp. water). This specificity may appear a drawback at first sight, but surfactants are in fact already used in the large majority of digital microfluidic applications, and the enormous panel of surfactants available offers easy and affordable combinations.

In conclusion, from a practical standpoint, our new optical approach may prove an elegant contactless alternative to microfabrication-intensive approaches such as resistive [51] or electrocapillary [27] methods.

\section{ACKNOWLEDGMENTS}

The authors wish to thank Nicolas Bertin and Alexandre Vilquin for early-stage experiments, and Isabelle Cantat and Benjamin Dollet for discussions. This work was partly supported by the CNRS and the Conseil régional d'Aquitaine through Grant No. 20061102030. The authors would also like to acknowledge networking support by the COST Action MP1205 (http://costmp1205.eu).
[1] J. Eggers and E. Villermaux, "Physics of liquid jets," Rep. Prog. Phys. 71, 036601 (2008).

[2] F. Savart, "Mémoire sur la constitution des veines liquides lancées par des orifices circulaires en mince paroi," Ann. Chim. Phys. 53, 337-398 (1833).

[3] J. Plateau, Statique expérimentale et théorique des liquides soumis aux seules forces moléculaires (GauthierVillars, Paris, 1873).

[4] J. W. Strutt, Lord Rayleigh, "On the instability of jets," Proc. London Math. Soc. s1-10, 4-13 (1878).

[5] J. W. Strutt, Lord Rayleigh, "On the capillary phenomena of jets," Proc. R. Soc. Lond. 29, 71-97 (1879).

[6] J. W. Strutt, Lord Rayleigh, "On the instability of a cylinder of viscous liquid under capillary force," Phil. Mag. 34, 145-154 (1892).

[7] A. Frischknecht, "Stability of cylindrical domains in phase-separating binary fluids in shear flow," Phys. Rev. E 58, 3495-3514 (1998).

[8] Y. Son, N. S. Martys, J. G. Hagedorn, and K. B. Migler, "Suppression of capillary instability of a polymeric thread via parallel plate confinement," Macro- molecules 36, 5825-5833 (2003).

[9] K. J. Humphry, A. Ajdari, A. Fernández-Nieves, H. A. Stone, and D. A. Weitz, "Suppression of instabilities in multiphase flow by geometric confinement," Phys. Rev. E 79, 056310 (2009).

[10] P. Guillot, A. Colin, and A. Ajdari, "Stability of a jet in confined pressure-driven biphasic flows at low Reynolds number in various geometries," Phys. Rev. E 78, 016307 (2008).

[11] M. San Miguel, M. Grant, and J. D. Gunton, "Phase separation in two-dimensional binary fluids," Phys. Rev. A 31, 1001-1005 (1985).

[12] R. Seemann, M. Brinkmann, T. Pfohl, and S. Herminghaus, "Droplet based microfluidics," Rep. Prog. Phys. 75, 016601 (2012).

[13] Dongqing Li (Editor), Encyclopedia of Microfluidics and Nanofluidics (Springer, New York, 2015).

[14] M. S. Brown, C. Fr. Brasz, Y. Ventikos, and C. B. Arnold, "Impulsively actuated jets from thin liquid films for high-resolution printing applications," J. Fluid Mech. 709, 341-370 (2012). 
[15] G. F. Christopher and S. L. Anna, "Microfluidic methods for generating continuous droplet streams," J. Phys. D: Appl. Phys. 40, R319 (2007).

[16] M. De Menech, P. Garstecki, F. Jousse, and H. A. Stone, "Transition from squeezing to dripping in a microfluidic t-shaped junction," J. Fluid Mech. 595, 141-161 (2008).

[17] Wingki Lee, Lynn M. Walker, and Shelley L. Anna, "Role of geometry and fluid properties in droplet and thread formation processes in planar flow focusing," Phys. Fluids 21, 032103 (2009).

[18] M. Robert de Saint Vincent, S. Cassagnère, J. Plantard, and J.-P. Delville, "Real-time droplet caliper for digital microfluidics," Microfluid. Nanofluid. 13, 261-271 (2012).

[19] H. Willaime, V. Barbier, L. Kloul, S. Maine, and P. Tabeling, "Arnold tongues in a microfluidic drop emitter," Phys. Rev. Lett. 96, 054501 (2006).

[20] Jae-Hoon Choi, Seung-Kon Lee, Jong-Min Lim, SeungMan Yang, and Gi-Ra Yi, "Designed pneumatic valve actuators for controlled droplet breakup and generation," Lab Chip 10, 456-461 (2010).

[21] Darren R. Link, Erwan Grasland-Mongrain, Agnès Duri, Flavie Sarrazin, Zhengdong Cheng, Galder Cristobal, Manuel Marquez, and David A. Weitz, "Electric control of droplets in microfluidic devices," Angew. Chem. Int. Ed. 45, 2556-2560 (2006).

[22] M. Belloul, J.-F. Bartolo, B. Ziraoui, F. Coldren, V. Taly, and A. I. El Abed, "High-throughput formation and control of monodisperse liquid crystals droplets driven by an alternating current electric field in a microfluidic device," Appl. Phys. Lett. 103, 033112 (2013).

[23] Yin Nee Cheung and Huihe Qiu, "Characterization of acoustic droplet formation in a microfluidic flow-focusing device," Phys. Rev. E 84, 066310 (2011).

[24] Lothar Schmid and Thomas Franke, "Saw-controlled drop size for flow focusing," Lab Chip 13, 1691-1694 (2013).

[25] Sung-Yong Park, Ting-Hsiang Wu, Yue Chen, Michael A. Teitell, and Pei-Yu Chiou, "High-speed droplet generation on demand driven by pulse laser-induced cavitation," Lab Chip 11, 1010-1012 (2011).

[26] Antoine Diguet, Hao Li, Nicolas Queyriaux, Yong Chen, and Damien Baigl, "Photoreversible fragmentation of a liquid interface for micro-droplet generation by light actuation," Lab Chip 11, 2666-2669 (2011).

[27] P. He, H. Kim, D. Luo, M. Marquez, and Z. Cheng, "Low-frequency ac electro-flow-focusing microfluidic emulsification," Appl. Phys. Lett. 96, 174103 (2010).

[28] V. G. Levich and V. S. Krylov, "Surface-tension-driven phenomena," Annu. Rev. Fluid Mech. 1, 293-316 (1969).

[29] H. Chraïbi and J.-P. Delville, "Thermocapillary flows and interface deformations produced by localized laser heating in confined environment," Phys. Fluids 24, 032102 (2012)

[30] J. C. Loulergue, P. Manneville, and Y. Pomeau, "Interface deflections induced by the Marangoni effect: an application to infrared-visible image conversion," J. Phys. D: Appl. Phys. 14, 1967 (1981).

[31] E. P. Furlani, "Temporal instability of viscous liquid microjets with spatially varying surface tension," J. Phys. A: Math. Gen. 38, 263 (2005).

[32] N. M. Nahas and R. L. Panton, "Control of surface tension flows: instability of a liquid jet," J. Fluids Eng. 112, 296-301 (1990).
[33] M. L. Cordero, F. Gallaire, and C. N. Baroud, "Quantitative analysis of the dripping and jetting regimes in coflowing capillary jets," Phys. Fluids 23, 094111 (2011).

[34] M. L. Cordero, É. Verneuil, F. Gallaire, and C. N. Baroud, "Time-resolved temperature rise in a thin liquid film due to laser absorption," Phys. Rev. E 79, 011201 (2009).

[35] M. Robert de Saint Vincent and J.-P. Delville, "Thermocapillary migration in small-scale temperature gradients: Application to optofluidic drop dispensing," Phys. Rev. E 85, 026310 (2012).

[36] C. Clanet and J. C. Lasheras, "Transition from dripping to jetting," J. Fluid Mech. 383, 307-326 (1999).

[37] B. Ambravaneswaran, S. D. Phillips, and O. A. Basaran, "Theoretical analysis of a dripping faucet," Phys. Rev. Lett. 85, 5332-5335 (2000).

[38] I. Marginean, P. Nemes, and A. Vertes, "Order-chaosorder transitions in electrosprays: The electrified dripping faucet," Phys. Rev. Lett. 97, 064502 (2006).

[39] P. Garstecki, M. J. Fuerstman, and G. M. Whitesides, "Nonlinear dynamics of a flow-focusing bubble generator: An inverted dripping faucet," Phys. Rev. Lett. 94, 234502 (2005).

[40] M. Hashimoto and G. M. Whitesides, "Formation of bubbles in a multisection flow-focusing junction," Small $\mathbf{6}$, 1051-1059 (2010).

[41] K. Nguyen, C. S. Daw, P. Chakka, M. Cheng, D. D. Bruns, C. E. A. Finney, and M. B. Kennell, "Spatiotemporal dynamics in a train of rising bubbles," Chem. Eng. J. 64, 191-197 (1996).

[42] A. Schmit, L. Salkin, L. Courbin, and P. Panizza, "Cooperative breakups induced by drop-to-drop interactions in one-dimensional flows of drops against micro-obstacles," Soft Matter 11, 2454-2460 (2015).

[43] D. C. Duffy, J. C. McDonald, O. J. A. Schueller, and G. M. Whitesides, "Rapid prototyping of microfluidic systems in poly(dimethylsiloxane)," Anal. Chem. 70, 4974-4984 (1998).

[44] M. Robert de Saint Vincent, J. Petit, M. Aytouna, J.-P. Delville, D. Bonn, and H. Kellay, "Dynamic interfacial tension effects in the rupture of liquid necks," J. Fluid Mech. 692, 499-510 (2012).

[45] Z. Khattari, P. Steffen, and T. M. Fischer, "Migration of a droplet in a liquid: effect of insoluble surfactants and thermal gradient," J. Phys.: Condens. Matter 14, 4823 (2002).

[46] B. Dollet, W. van Hoeve, J.-P. Raven, P. Marmottant, and M. Versluis, "Role of the channel geometry on the bubble pinch-off in flow-focusing devices," Phys. Rev. Lett. 100, 034504 (2008).

[47] P. Guillot and A. Colin, "Stability of parallel flows in a microchannel after a T junction," Phys. Rev. E 72, 066301 (2005).

[48] H. Chraïbi, D. Lasseux, R. Wunenburger, É. Arquis, and J.-P. Delville, "Optohydrodynamics of soft fluid interfaces: Optical and viscous nonlinear effects," Eur. Phys. J. E 32, 43-52 (2010).

[49] D. J. Sanders, "Temperature distributions produced by scanning Gaussian laser beams," Appl. Opt. 23, 30-35 (1984).

[50] J. N. Israelachvili, Intermolecular and Surface Forces (Third Edition) (Academic Press, San Diego, 2011).

[51] J. M. Chwalek, D. P. Trauernicht, C. N. Delametter, 
R. Sharma, D. L. Jeanmaire, C. N. Anagnostopoulos, G. A. Hawkins, B. Ambravaneswaran, J. C. Pandi- taratne, and O. A. Basaran, "A new method for deflecting liquid microjets," Phys. Fluids 14, L37-L40 (2002). 